\title{
An FPGA-Based Quench Detection and Continuous Logging System for Testing Superconducting Magnets
}

\author{
R. Pilipenko, R. H. Carcagno, A. Makulski, R. Nehring, D. F. Orris
}

\begin{abstract}
A second generation quench detection system with two concurrent data logging modes was developed at Fermilab for testing superconducting magnets. The system consists of two functional components: An active quench detector based on a reconfigurable input/output module with an onboard fieldprogrammable gate array (FPGA); and a data logger based on a set of simultaneous sampling ADC modules. The data logger has two concurrent modes of operation: A fast mode that is triggered to capture a user specified window of data at rates up to $10 \mathrm{kHz}$; and a continuous mode that can log data at rates between $0.1 \mathrm{~Hz}$ and $100 \mathrm{~Hz}$ continuously using the same data loggers. The modular design uses commercially available DAQ hardware in combination with programmable isolation amplifiers developed in-house. This approach allows the new system to be easily scaled to handle multiple magnets or magnets with more complex coil and lead voltage tap configurations. The new system has been used to test the MICE Spectrometer Solenoid. A detailed description of the system along with test results is presented in this paper.
\end{abstract}

Index Terms - Instrumentation and controls, quench detection, superconducting magnets

\section{INTRODUCTION}

$\mathrm{T}$ he Magnet Test Facility (MTF) at Fermilab has employed quench detection and protection systems used for more than two decades[1]-[5]. In 2005, MTF designed and implemented the first FPGA-based quench detection system [5]. Firmware running on the FPGA detects the resistive voltages produced when the superconducting coil goes normal (quenches). When a quench is detected, protection logic, also implemented in the FPGA, switches a dump resistor into the circuit and ramps the magnet down to zero current. The detector also triggers fast data loggers to capture coil voltages in a window surrounding the quench event for offline analysis.

The flexible FPGA-based system allows a wide variety of magnets with a broad spectrum of characteristics to be tested easily. Additional functionality was needed, however, to capture data during the several hours required to energize magnets with very large inductances. Two examples of such magnets are the MICE Spectrometer Solenoid [8], and the

Manuscript received October 9, 2012. This work was supported by the U.S. Department of Energy.

The authors are with the Fermi National Accelerator Laboratory, PO Box 500, Batavia, IL 60510 USA, pilipen@fnal.gov.

\section{MICE Coupling Coil [9].}

Hardware improvements were made to increase the flexibility of the system and to reduce the time required to setup individual magnets. Furthermore the second generation system continuously monitors and logs voltage tap signals during magnet energization in addition to capturing tap data in a time window around a quench.

\section{HARDWARE MODIFICATIONS}

Even though the majority of magnets tested at MTF require some device specific re-configuration of the quench detection system hardware, the fundamental building blocks do not change. The important subsystems are shown in Fig. 1: the voltage tap interface, isolation amplifiers, filters, the quench management interface, and the data acquisition and processing subsystem.

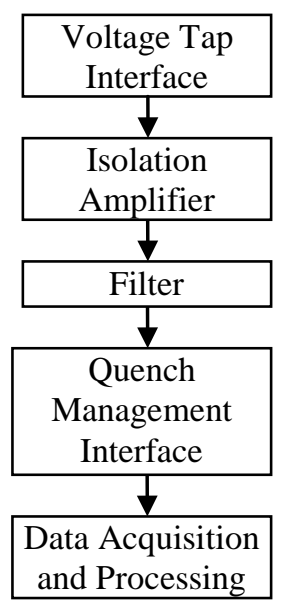

Fig. 1. Quench Detection System Block Diagram

The voltage tap interface allows signal connections from taps in the magnet coils and power leads to the isolation amplifiers to be easily reconfigured. The isolation amplifiers provide isolation between magnet voltage taps and all other equipment, and scale the signals to levels appropriate for digitization by the ADCs. The gains of the isolation amplifiers and the response of the filters they drive can be set to accommodate a wide range of magnets. The quench management interface relays the scaled and filtered tap signals to the data acquisition and processing subsystem. This interface also manages auxiliary signals for all magnet specific 
devices, such as heaters, external quench indicators, etc. The data acquisition and processing, the core of system, digitizes and processes the signals.

The quench detection rack shown in Fig. 2. The voltage tap interface, isolation amplifiers, filters, and the quench management interface were built in-house, while the data acquisition and processor are commercial sourced PXI units.

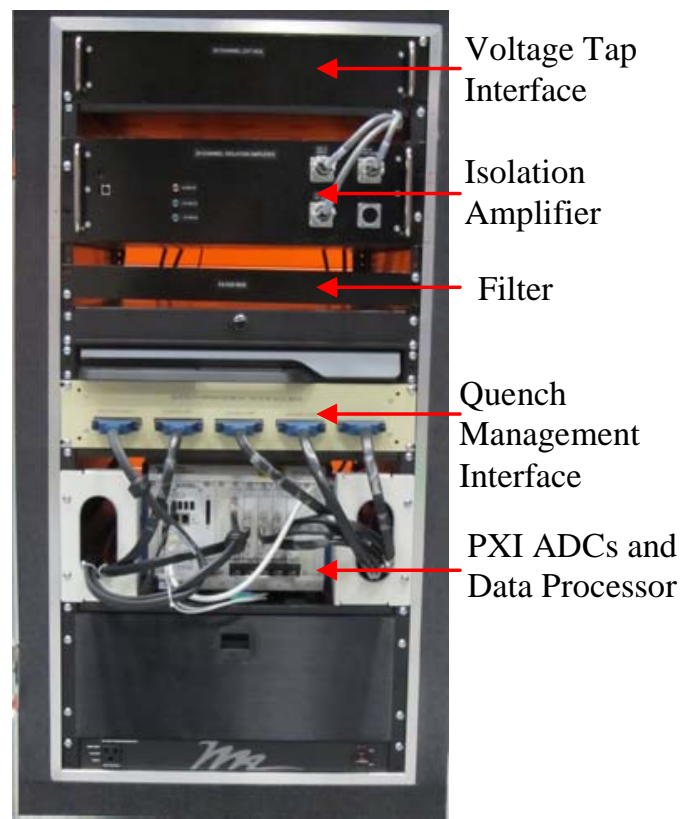

Fig. 2. FPGA-Based Quench Detection and Continuous Logging System

Although much of the same hardware was used in the first generation system, several hardware components have been upgraded. The isolation amplifiers were originally designed such that the gains of each channel could be programmed by either manually by changing setting jumpers or via a Serial Peripheral Interface (SPI). The first generation system, however, did not utilize the SPI interface. All isolation amplifier gains can now be controlled programmatically.

The filter module was redesigned from the ground up. While previously fixed band-width filters employed and changing filter characteristics required physically changing the unit, pass-bands in the replacement system can be set using switches.

\section{SOFTWARE MODIFICATIONS}

Significant improvements have been made to the software of the upgraded quench detection system. The firmware/software of the system consists of two major components: an active quench detector, and the data logger. The active quench detection component is implemented as firmware running on a NI PXI-7833R FPGA module. It performs voltage tap signal digitization, filtering, detection and triggering. The data logger uses an NI PXI-6143 module to record tap signals in the event of quench. Both modules are commercial PXI form-factor devices, manufactured by National Instrument (NI) similar to those used in the first generation system. For the second generation system, an entirely new data logger with two concurrent modes of operation, fast and continuous, was designed and implemented.

In fast mode, signals digitized by the ADCs are stored in a circular buffer located in PXI controller memory. When quench event is detected, the pre and post-trigger data in the circular buffer is recorded. Up to 32 active channels at rates of up to $10 \mathrm{kHz}$ can be recorded during operation in fast mode.

The data stored in the circular buffer is also used by the continuous logger. The data in the buffer may be either subsampled or averaged to reduce the sampling rate prior to recording (see Fig. 3). Continuous data from 32 channels can be logged at sampling rates from as low as $0.1 \mathrm{~Hz}$ up to 100 $\mathrm{Hz}$. Up to 24 hours of continuous data has been recorded at the rate of $5 \mathrm{~Hz}$ typically used.

Other software components of the quench detection system software include: isolation amplifier configuration, a data visualization program; and a quench analysis program. The isolation amplifier configuration and data visualization programs are Labview applications while the quench analysis program is an executable compiled from Matlab source code. As part of the upgrade, the quench analysis program was modified to accommodate data from both the fast and continuous loggers.

\section{TEST RESULTS}

The new system was used to test the MICE Spectrometer Solenoid at Livermore, CA. The system was successfully integrated with other MICE test equipment and was used to monitor coil and lead tap signals during magnet energization; to detect quenches; to monitor and record of voltage taps signals prior to, during and after quench events. The MICE magnet is protected by a passive protection system [6], [7] but the quench detection system shut down the magnet power supplies when a quench was detected.

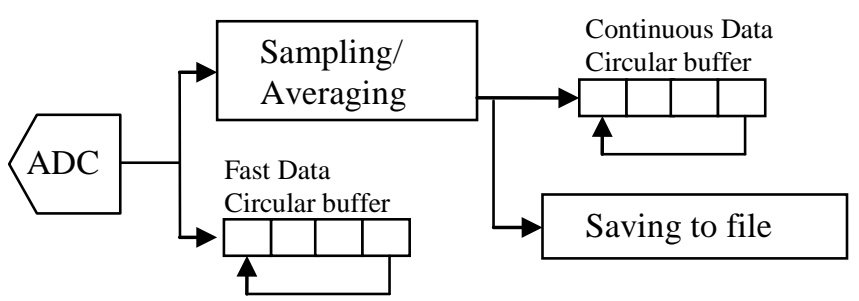

Fig. 3. Data flow for Fast and Continuous data logger modes;

Fig. 4 shows data from some of the 32 channels logged simultaneously at $3 \mathrm{~S} / \mathrm{s}$ during a 2.7 hour ramp of the MICE Spectrometer Solenoid. Fig. 5 shows 30 seconds of fast 
logger data recorded at $3571 \mathrm{~S} / \mathrm{s}$ during a quench and the subsequent dissipation of the stored energy in end coils E2 [7].

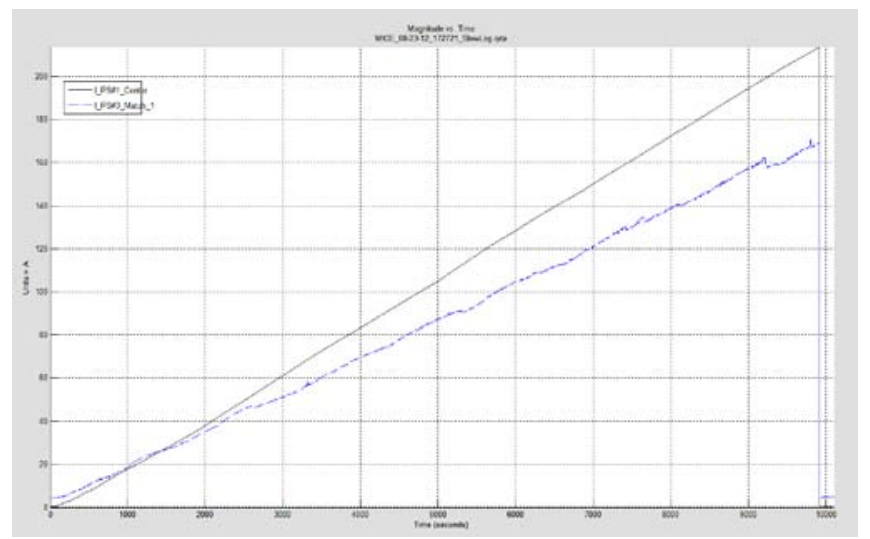

Fig. 4. Example of data acquired during 2.7 hours current ramp

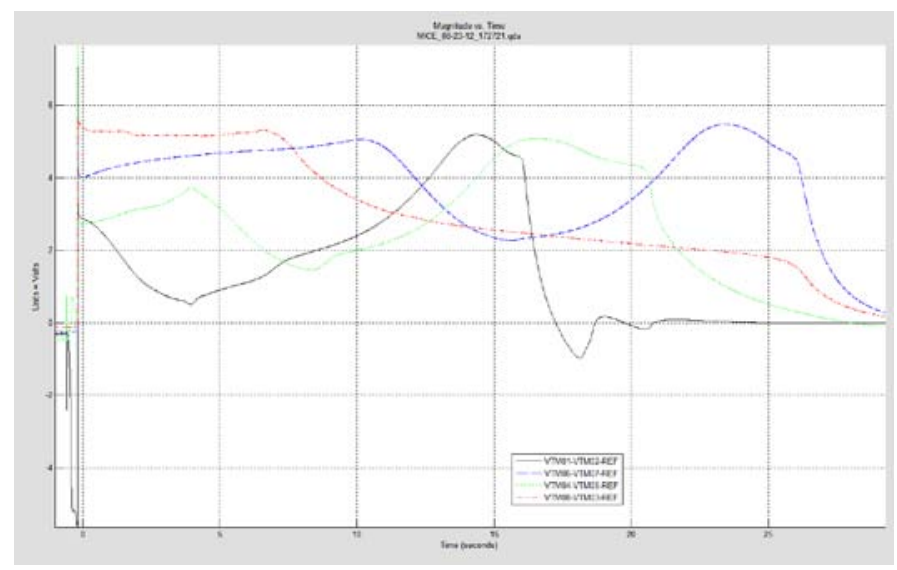

Fig. 5. Quench on VTM01-VTM02 Coil, and following energy dissipation process

\section{CONCLUSION}

The second generation quench detection system developed by the Fermilab MTF includes continuous data logging of signal for periods of up to 24 hours to facilitate testing of magnets with large inductances. The system also incorporates other upgrades to improve system flexibility and operational efficiency, such as fully programmable gains for all isolation amplifiers. The system has been successfully used to detect quenches and log fast and continuous data during testing of the MICE solenoid in Livermore. The new features will be incorporated in for the third generation of quench detection systems currently under development for the Fermilab Solenoid Test Facility.

\section{ACKNOWLEDGMENT}

The authors thank Electrical Technical Support group for their work in quench detection system hardware assembling.

\section{REFERENCES}

[1] M. J. Lamm, J. DiMarco, E. Desavouret, S. Feher, J.D. Garvey, C. Hess, P.J. Limon, J.M. Nogiec, D. F. Orris, J. Pachnik, T. Peterson, S. Sharonov, J.B. Strait, C. Sylvester, J.W. Sim, M. Tartaglia, J.C. Tompkins, A.V. Zlobin, "A new facility to test superconducting accelerator magnets,” in Proc. 1997 Particle Accelerator Conf., vol.3, Vancouver, pp.3395-3397.

[2] D. F. Orris, S. Feher, M. J. Lamm, S. Sharonov, M. Tartaglia, “A digital quench detection system for superconducting magnets," in Proc. 1999 Particle Accelerator Conf., New York, pp. 3191-3193.

[3] D.F. Orris, R. Carcagno, "A modular and extensible data acquisition and control system for testing superconducting magnets," in Proc. 2001 Particle Accelerator Conf., Chicago, IL, pp.3463-3465.

[4] D. F. Orris, R. Carcagno, S. Feher, M. J. Lamm,J. Nogiec, P. Schlabach, M. Tartaglia, and J. C. Tompkins, "A quench management system for testing superconducting magnets," IEEE Trans. Appl. Supercond., vol. 13, no. 2, pp. 1700-1703, Jun. 2003.

[5] R. H. Carcagno, S. Feher, M. Lamm, A. Makulski, R. Nehring, D. F. Orris, Y. Pischalnikov, M. Tartaglia, "An FPGA-based quench detection and protection system for superconducting accelerator magnets," in Proc. 2005 Particle Accelerator Conf., Knoxville, TN, pp. 3502-3504.

[6] M. A. Green, "A Design of a Rapid Discharge Varistor System for the MICE Magnet Circuits”, LBNL, Berkeley, CA, Rep. LBNL-900E, 2008.

[7] S. P. Virostek and M. A. Green, "The results of Tests of the MICE spectrometer solenoid,” IEEE Trans. Appl. Supercond., vol. 20, no. 3, pp. 377-380, Jun. 2010.

[8] M. A. Green and S. P. Virostek, "The Dimensions and Number of Turns for the Tracker Solenoids As-Built compared to the Original Magnet Design”, LBNL, Berkley, CA, Rep. LBNL-899E, 2008.

[9] L. Wang, F. Y. Xu, H. Wu, X. K. Liu, L. K. Li, X. L. Guo, H. Pan, A. B. Chen, M. A. Green, D. R. Li and S. P. Virostek, "Magnetic and Cryogenic Design of MICE Coupling Solenoid Magnet System," IEEE Trans. Appl. Supercond., vol. 19, no. 3, pp. 1344-1347, Jun. 2009. 